\title{
A Decomposition Theorem and Its Implications to the Design and Realization of Two-Dimensional Filters
}

\author{
ANASTASIOS N. VENETSANOPOULOS, SENIOR MEMBER, IEEe, AND BASIL G. MERTZIOS, MEMBER, IEEE
}

\begin{abstract}
It is shown that an arbitrary rational 2-D transfer function can be expanded in first order terms, each one of which is a function of only one of the two variables. This method leads naturally to reconfigurable filters with great modularity and parallelism, which can realize any rational transfer function up to a given order.
\end{abstract}

\section{INTRODUCTION}

$\mathrm{T}$ HE most serious problem in the generalization of 1-D (one-dimensional) digital filtering techniques to 2-D (two-dimensional) is the fact that there is no fundamental theorem of algebra for polynomials in two independent variables. Factorization of $1-D$ polynomials into products of first order terms plays a fundamental role in the development of stability tests and stabilization, filter design algorithms and implementation schemes, such as parallel and cascade structures. Huang, Schreiber, and Tretiak [1] called the lack of the corresponding theorem "a fundamental curse" of 2-D filtering. Bliss [2] mentioned that "a two variable polynomial is not in general factorable into first order polynomials; rather a two-variable polynomial can be factored into irreducible factors, which are themselves two variable polynomials, but which cannot be further factored."

During the last few years considerable effort has been directed by a number of authors to establish algorithms and conditions for factorization [3]-[13]. Chakrabarti et $a l$. in [3] treated the cases where two variable polynomials can be expressed as a product or sum of special types of two variable polynomials of lower order, and in [4] presented an algorithm to determine the reducibility or the irreducibility of any arbitrary multivariable polynomial with integer coefficients. In [5] the decomposability of 2D transfer functions into 1-D components was considered. Morf et al. [6] proposed an algorithm for the case of primitive factorization (i.e., in the case that one factor is a function of one variable only and the other is a function of both variables), and Bose [7], [8] gave a criterion to determine whether any two multivariable polynomials are relatively prime and an algorithm for the extraction of the greatest common factor. In [9] and [10], feedback was used for the separation of some transfer functions into

Máruscript received July 14, 1983; revised November 19, 1984. This work was supported by the Natural Sciences and Engineering Research Council of Canada under Grant A7397.

A. N. Venetsanopoulos is with the Department of Electrical Engineering, University of Toronto, Toronto, Ont., Canada M5S 1A4.

B. G. Mertzios is with the Department of Electrical Engineering, Democritus University of Thrace, Greece. terms which are functions of one of the two variables. Treitel and Shanks in [11] applied an approximation technique to the expansion of an arbitrary planar filter impulse response into a converging sum of individually separable 2-D filters. Ekstrom and Woods [13] presented an alternate technique, the spectral factorization approach, which allows the factorization of 2-D transfer functions into terms with specified regions of analyticity. Their approach is based on decompositions of the complex cepstrum.

While most earlier contributions were either special cases or approximations to the general decomposition problem, in this paper we propose a method for the exact decomposition of a general 2-D real rational transfer function in first order terms, each one of which is a function of only one of the two variables. The motivation behind this approach is to give a general realization method, which possesses a high degree of modularity.

Recent development of VLSI techniques have resulted in enormous possibilities for the realization and implementation of sophisticated algorithms of high complexity. Since it is known that the most advantageous configurations are parallel and cascade forms, consisting of second order terms and real coefficients [14], we choose to combine complex first order terms with appropriate conjugate terms, in order to achieve second order terms with real coefficients.

Present tendencies for the reduction of the cost of hardware, coupled with an increase of the complexity of implementation algorithms and applications, indicate that considerations leading to moderate savings of dynamic elements (registers, adders, multipliers, etc.) are not as meaningful as they were in the past. Other considerations, such as modularity, parallelism, regularity, flexibility, and generality are of paramount importance and to that end realizations exhibiting these properties become desirable.

The proposed method can be seen as a decomposition, a special case of which, in the case of 1-D polynomials, is simple factorization. While the fundamental theorem of algebra expands a given polynomial of one variable into a product of first order terms, the proposed method makes use of array expansions to provide the additional degrees of freedom required for the expansion of a polynomial of two variables. Inasmuch as it is general, the method can be used for the construction of general adaptive and reconfigurable filters. Such filters possess modular form and are able to realize any rational transfer function up to a given order, by varying the values of a set of parameters. 


\section{A General Decomposition of a 2-D Transfer FUNCTION}

In this section we shall establish a theorem to express an arbitrary 2-D transfer function by first order terms of one variable only.

Theorem 1: The 2-D rational transfer function of the general form

$$
H\left(z_{1}, z_{2}\right)=\frac{q\left(z_{1}, z_{2}\right)}{p\left(z_{1}, z_{2}\right)}=\frac{\sum_{i=0}^{n_{1}} \sum_{j=0}^{m_{1}} q_{i j} z_{1}^{i} z_{2}^{j}}{\sum_{i=0}^{n_{2}} \sum_{j=0}^{m_{2}} p_{i j} z_{1}^{i} z_{2}^{j}}
$$

can be expressed by terms of the form

$$
\left(z_{1}-z_{1 i}\right), \quad\left(z_{2}-z_{2 j}\right)
$$

only, where $z_{1 i}, z_{2 j}$ are constants and $i=1,2, \cdots, \max$ $\left(n_{1}, n_{2}\right), j=1,2, \cdots, \max \left(m_{1}, m_{2}\right)$.

Proof: In the following, one of the possible proofs will be given.

a) Numerator.

The polynomial $q\left(z_{1}, z_{2}\right)$ can be written in the form

$$
q\left(z_{1}, z_{2}\right)=Z_{1}^{T} Q Z_{2}
$$

where

$$
\begin{aligned}
\boldsymbol{Z}_{1}=\left[\begin{array}{c}
1 \\
z_{1} \\
\vdots \\
z_{1}^{n_{1}}
\end{array}\right], \boldsymbol{Z}_{2}=\left[\begin{array}{c}
1 \\
z_{2} \\
\vdots \\
z_{2}^{m_{1}}
\end{array}\right], \\
\boldsymbol{Q}=\left[\begin{array}{cccc}
q_{00} & q_{01} & \cdots & q_{0 m_{1}} \\
q_{10} & q_{11} & \cdots & q_{1 m_{1}} \\
\vdots & \vdots & & \vdots \\
q_{n_{1} 0} & q_{n_{1} 1} & \cdots & q_{n_{1} m_{1}}
\end{array}\right] .
\end{aligned}
$$

The polynomial (2) can be written equivalently as

$$
q\left(z_{1}, z_{2}\right)=\boldsymbol{Z}_{1}^{T} \boldsymbol{R S Z _ { 2 }}
$$

by writing the matrix $Q$ as a product of two other matrices $\boldsymbol{R}, \boldsymbol{S}$. The matrix $\boldsymbol{R}$ might be chosen arbitrarily as a nonsingular $\left(n_{1}+1\right) \times\left(n_{1}+1\right)$ matrix. In this case the matrix $S$ is determined by

$$
S=R^{-1} Q
$$

and has dimensions $\left(n_{1}+1\right) \times\left(m_{1}+1\right)$.

We can readily see that

$$
\begin{aligned}
\boldsymbol{Z}_{1}^{T} \boldsymbol{R} & =\left[r_{0}\left(z_{1}\right), \cdots, r_{i}\left(z_{1}\right), \cdots, r_{n_{1}}\left(z_{1}\right)\right] \\
\boldsymbol{S} \boldsymbol{Z}_{2} & =\left[s_{0}\left(z_{2}\right), \cdots, s_{i}\left(z_{2}\right), \cdots, s_{n_{1}}\left(z_{2}\right)\right]^{T}
\end{aligned}
$$

where

$$
\begin{gathered}
r_{i}\left(z_{1}\right)=r_{0 i}+r_{1 i} z_{1}+\cdots+r_{j i} z_{1}^{j}+\cdots r_{n i} z_{1}^{n_{1}}, \\
i=0,1, \cdots, n_{1}
\end{gathered}
$$

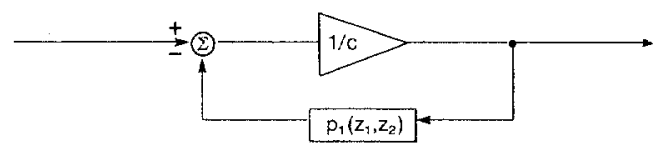

Fig. 1. Transfer function of $H_{D}\left(z_{1}, z_{2}\right)=1 / p\left(z_{1}, z_{2}\right)$.

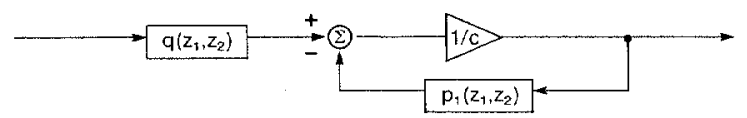

Fig. 2. The feedback representation of the total transfer function.

$$
\begin{gathered}
s_{i}\left(z_{2}\right)=s_{i 0}+s_{i 1} z_{2}+\cdots+s_{i j} z_{2}^{j}+\cdots+s_{i m_{1}} z_{2}^{m_{i}}, \\
i=0,1, \cdots, n_{1}
\end{gathered}
$$

Making use of (5) and (6), the polynomial (2) may be written as

$$
\begin{aligned}
q\left(z_{1}, z_{2}\right)= & {\left[r_{0}\left(z_{1}\right), \cdots, r_{i}\left(z_{1}\right), \cdots, r_{n 1}\left(z_{1}\right)\right] } \\
& \cdot\left[s_{0}\left(z_{2}\right), \cdots, s_{i}\left(z_{2}\right), \cdots, s_{n 1}\left(z_{2}\right)\left[^{T}\right.\right. \\
= & \sum_{i=0}^{n_{1}} r_{i}\left(z_{1}\right) s_{i}\left(z_{2}\right) .
\end{aligned}
$$

Obviously, the polynomials $r_{i}\left(z_{1}\right)$, and $s_{i}\left(z_{2}\right)$ can be expressed as products of first order terms. We therefore conclude that $q\left(z_{1}, z_{2}\right)$ can be expressed as a sum of products of first order terms, each one of which is a function of only one of the two variables.

b) Denominator.

We write the denominator of the given transfer function as follows

$$
p\left(z_{1}, z_{2}\right)=c+p_{1}\left(z_{1}, z_{2}\right)
$$

where the polynomial $p_{1}\left(z_{1}, z_{2}\right)$ does not contain a constant term. If we choose the constant $1 / c$ in the forward branch and the polynomial $p_{1}\left(z_{1}, z_{2}\right)$ in the feedback branch, we obtain the configuration

$$
H_{D}\left(z_{1}, z_{2}\right)=\frac{1 / c}{1+\frac{1}{c} p_{1}\left(z_{1}, z_{2}\right)}
$$

shown in Fig. 1.

Finally using the same technique used for the numerator, we may write $p_{1}\left(z_{1}, z_{2}\right)$ in the form of an array (8).

The representation of the total transfer function is given in Fig. 2. Note that the proof of Theorem 1 is just one possible proof and does not necessarily lead to the most economical and advantageous realization of the filter.

Lemma: Any physical realization of an arbitrary 2-D rational transfer function $H\left(z_{1}, z_{2}\right)$, given by (1), requires a feedback configuration whose path transmittance in the feedback branch is a polynomial in both $z_{1}, z_{2}$.

Proof: Assume that $H\left(z_{1}, z_{2}\right)$ can be realized by a general lattice of terms of order one, in each one of the two variables. Such a lattice does not contain feedback in both 
variables. Therefore, $H\left(z_{1}, z_{2}\right)$ is of the form

$$
H\left(z_{1}, z_{2}\right)=\sum_{i=1}^{L} H_{i 1}, \cdots, H_{i j}, \cdots, H_{i M}
$$

where $H_{i j}$ are factors, each one of which is a numerator or denominator term in one of the two variables only. In this case, the denominator of the resulting transfer function (11) is separable, which contradicts our hypothesis.

Q.E.D.

Note that ordinary factorization does not apply in general to a function of the form (1), because it lacks the necessary degrees of freedom required to represent this function. The proposed decomposition provides the necessary degrees of freedom to guarantee decomposition.

The realization proposed exhibits a high degree of parallelism with parallel branches which are separable. It is shown in Section III that each of its parallel branches can operate simultaneously on the input, resulting to a substantial increase in data throughout.

Theorem 1 can be easily extended to apply to half-plane filters of the form [13]

$$
H\left(z_{1}, z_{2}\right)=\frac{q\left(z_{1}, z_{2}\right)}{p\left(z_{1}, z_{2}\right)}=\frac{\sum_{i=0}^{n_{1}} \sum_{j=-m_{1}}^{m_{1}} q_{i j} z_{1}^{i} z_{2}^{j}}{\sum_{i=0}^{n_{2}} \sum_{j=-m_{2}}^{m_{2}} p_{i j} z_{1}^{i} z_{2}^{j}} .
$$

In this case the transfer function $H\left(z_{1}, z_{2}\right)$ can be expressed by terms of the form

$$
\left(z_{1}-z_{1 i}\right), \quad\left(z_{2}^{l}-z_{2 j}\right), \quad \text { where } l=1,-1 .
$$

The proof is similar to that of the quarter-plane case.

We note that in special cases, a 2-D rational transfer function can be realized with forward and feedback branches with transmittance only in $z_{1}$ or $z_{2}$. An illustrative example for this latter situation is the case where the given transfer function can be written in the form

$$
\begin{aligned}
H\left(z_{1}, z_{2}\right) & =\frac{q\left(z_{1}, z_{2}\right)}{p\left(z_{1}, z_{2}\right)}=\frac{t\left(z_{1}, z_{2}\right) s\left(z_{1}, z_{2}\right)}{1+r\left(z_{1}\right) s\left(z_{1}, z_{2}\right)} \\
& =\left[t\left(z_{1}, z_{2}\right)\right]\left[\frac{s\left(z_{1}, z_{2}\right)}{1+r_{1}\left(z_{1}\right) s\left(z_{1}, z_{2}\right)}\right] .
\end{aligned}
$$

The above transfer function can be realized by an array representing $t\left(z_{1}, z_{2}\right)$, in cascade with a feedback loop having $s\left(z_{1}, z_{2}\right)$ in the forward branch and $r\left(z_{1}\right)$ in the feedback branch. However, since in the general case a 2-D polynomial cannot be factored in lower order $1-D$ and/or 2-D polynomials, the above is not the general case.

Clearly, the decomposition procedure used in the proof of Theorem 1 is not unique. A generalization of this latter decomposition is given by the following expression

$$
q\left(z_{1}, z_{2}\right)=Z_{1}^{T} R \boldsymbol{R}^{-1} \boldsymbol{Q S}{ }^{-1} \boldsymbol{S} \boldsymbol{Z}_{2}
$$

where only one of the two matrices $R, S$ is taken into account every time. The auxiliary matrices $\boldsymbol{R}$ and $\boldsymbol{S}$ can be used in order to reduce the number of terms required for the realization. To this end $R$ is used if $m_{1}>n_{1}$ and $S$ if $m_{1}<n_{1}$.

Furthermore, a more economical decomposition can be obtained if the auxiliary matrix $\boldsymbol{R}$ in (3) is chosen such that the number of terms in the sum (8) is minimized. To this end, suppose now without loss of generality, that $n_{1}$ $\leq m_{1}$. Then rank $Q=r \leq n_{1}+1$ and a more economical realization can be obtained if a decomposition of the form

$$
q\left(z_{1}, z_{2}\right)=\left[Z_{1}^{T} R^{-1}\right]\left[R Q Z_{2}\right]
$$

is considered, where $\boldsymbol{R}$ is a nonsingular $\left(n_{1}+1\right) \times\left(n_{1}+\right.$ 1) matrix, chosen to satisfy the following relation

$$
\boldsymbol{R} \boldsymbol{Q}=\left[\frac{\hat{Q}}{\boldsymbol{O}}\right]
$$

where $\hat{Q}$ is an $r \times\left(n_{1}+1\right)$ matrix and $\boldsymbol{O}$ a null $\left(n_{1}+1\right.$ $-r) \times\left(n_{1}+1\right)$ matrix. Taking into account (16), (15) can be written as a sum of $r$ terms of the form (8).

For 1-D polynomials, the order, i.e., the highest degree of $z$ determines the available degrees of freedom. For 2-D polynomials we have two orders with respect to $z_{1}, z_{2}$, which however do not determine the available degrees of freedom. To be more specific, let the numerator polynomial $q\left(z_{1}, z_{2}\right)$ given in (2). Suppose that rank $Q=r \leq \min$ $\left(n_{1}+1, m_{1}+1\right)$ where $\boldsymbol{Q}$ is the corresponding coefficient matrix. Then the realization of this polynomial, based on the decomposition theorem, needs only $r$ parallel branches. Thus, the number of the coefficients in the decomposed form equals to $t=r\left[\left(n_{1}+1\right)+\left(m_{1}+1\right)\right]$, which is greater than or equal to the number of coefficients $\left(n_{1}+\right.$ 1) $\left(m_{1}+1\right)$ in the given form.

At this point we want to clarify the fact that the condition $\operatorname{rank} Q=r<\min \left(n_{1}+1, m_{1}+1\right)$ is not a sufficient one for the primitive factorization of the polynomial $q\left(z_{1}\right.$, $z_{2}$ ) as follows:

$$
q\left(z_{1}, z_{2}\right)=q_{1}\left(z_{1}\right) q_{2}\left(z_{2}\right) q_{3}\left(z_{1}, z_{2}\right) .
$$

Clearly, in the case of primitive factorization we have

$$
\operatorname{rank} Q=\operatorname{rank} Q_{3}=r \leq \min \left(\hat{n}_{1}+1, \hat{m}_{1}+1\right)
$$

where

$$
\hat{n}_{1} \leq n_{1}, \quad \hat{m}_{1} \leq m_{1}, \quad \text { and }\left(\hat{n}_{1}, \hat{m}_{1}\right)<\left(n_{1}, m_{1}\right) .
$$

Then the polynomial $q\left(z_{1}, z_{2}\right)$ needs for its implementation $r$ parallel branches with

$$
\begin{aligned}
\hat{t}= & r\left[\left(\hat{n}_{1}+1\right)+\left(\hat{m}_{1}+1\right)\right] \\
& +\left(n_{1}-\hat{n}_{1}\right)+\left(m_{1}-\hat{m}_{1}\right)
\end{aligned}
$$

coefficients, since $n_{1}-\hat{n}_{1}, m_{1}-\hat{m}_{1}$ are the orders of the polynomials $q_{1}\left(z_{1}\right), q_{2}\left(z_{2}\right)$, respectively. It can be readily seen that $t \geq \hat{t}$, where the equality holds for $r=1$, which is the case of exact factorization of the 2-D polynomial in $1-D$ terms. From the above results, the rank $Q=r$ determines the smallest necessary number of parallel branches for the realization of a $2-\mathrm{D}$ polynomial. To this end, the 
term "rank of decomposition" is proposed here to characterize the number $r$.

In the following, some specific realizations based on the decomposition theorem will be concisely reviewed. The main difference among them is the specific choice of the auxiliary matrices $R, S$.

\section{A. The Jordan Form Decomposition [15]}

A special form of Theorem 1 using the Jordan form is possible. This approach is based on matrix diagonalization. In the general case where $n_{1} \neq m_{1}$ (i.e., $\hat{Q}$ is not a square matrix), we consider the augmented square matrix $Q$, defined by

$$
\hat{Q}=[Q \mid O], \quad n_{1}>m_{1}
$$

where $\boldsymbol{O}$ is an $n_{1} \times\left(n_{1}-m_{1}\right)$ null matrix, or

$$
\hat{\boldsymbol{Q}}=\left[\frac{\boldsymbol{Q}}{\boldsymbol{O}}\right], \quad n_{1}<m_{1}
$$

where $\boldsymbol{O}$ is now an $\left(m_{1}-n_{1}\right) \times m_{1}$ null matrix. We may now write

$$
\hat{\boldsymbol{Q}}=\boldsymbol{H} \boldsymbol{J} \boldsymbol{H}^{-1}
$$

where $\boldsymbol{H}$ is the transforming matrix consisting of the eigenvectors of $\hat{Q}$, and $\boldsymbol{J}$ the Jordan matrix of $\hat{Q}$, consisting of the eigenvalues of $\hat{\boldsymbol{Q}}$. Thus, the Jordan form decomposition may be viewed as a special case of Theorem 1 . In this case, the augmented coefficient square matrix $\hat{Q}$ is written as a product of two matrices $R=H J$ and $S=$ $\boldsymbol{H}^{-1}$. Then

$$
q\left(z_{1}, z_{2}\right)=\sum_{i=0}^{l} a_{i}\left(z_{1}\right) b_{i}\left(z_{2}\right), \quad l=\max \left(n_{1}, m_{1}\right) .
$$

Note that the matrices $\boldsymbol{R}, \boldsymbol{S}$ are determined in terms of the coefficient matrix $Q$ and cannot be arbitrarily defined. Furthermore the number of parallel branches is the max $\left(n_{1}+1, m_{1}+1\right)$.

\section{B. The Singular Value Decomposition (SVD) [11], [16]}

In this case, the coefficient matrix $Q$ of the 2-D polynomial $q\left(z_{1}, z_{2}\right)$ is expressed as a product of the form

$$
Q=U[\Lambda]^{1 / 2} V^{t}
$$

where $\Lambda$ is a diagonal matrix whose entries are the singular values of $Q$, and $U$, and $V$ are the row and the column eigenvalue system of $Q$, respectively [16]. Due to the orthonormal nature of $\boldsymbol{U}$ and $\boldsymbol{V}, \boldsymbol{Q}$ can be written as

$$
Q=\sum_{i=1}^{r} \lambda_{i}^{1 / 2} \boldsymbol{u}_{i} \boldsymbol{v}_{i}^{t}, \quad r=\operatorname{rank} \boldsymbol{Q}
$$

where $\boldsymbol{u}_{i}$, and $\boldsymbol{v}_{i}$, and $\lambda_{i}$ are the column vectors of $\boldsymbol{U}, \boldsymbol{V}$ and diagonal terms of $\Lambda$, respectively. The SVD techniques have been used in the past for the approximation of a filter in terms of a number of separable filters [11] and for image processing applications [16]. The matrix $Q$ need not be square and the matrices $R, S$ can be identified by $U[\Lambda]^{1 / 2}, V^{t}$ or $U,[\Lambda]^{1 / 2} V^{t}$, respectively.

\section{The Lower-Upper Triangular, (LU) Decomposition [17]-[19]}

This is also a special case of the general decomposition scheme where the matrix $\boldsymbol{Q}$ is written as

$$
\boldsymbol{Q}=\boldsymbol{L} \boldsymbol{D} \boldsymbol{U}=\sum_{i=1}^{r} d_{i} \boldsymbol{l}_{i} \boldsymbol{u}_{i}
$$

where $\boldsymbol{L}, \boldsymbol{l}_{i}$ and $\boldsymbol{U}, \boldsymbol{u}_{i}$ are lower and upper triangular matrices, respectively, and $d_{i}$ scalars. As in the previous case, $R$ and $S$ can be identified by $L D, U$ or $L, D U$, respectively.

\section{Other Decompositions}

Furthermore, the matrix $Q$ in (2) could be substituted by $Q Q^{-} Q$, where " -" denotes one of the different generalized inverses. In this case, $\boldsymbol{R}$ and $\boldsymbol{S}$ can be identified by $Q, Q^{-} Q$ or $Q Q^{-}$and $Q$, respectively.

However, it should be mentioned here that desirable characteristics, such as roundoff noise reduction and reduction of the computational cost, can be obtained by using orthogonal auxiliary matrices (therefore having known inverses) with integer elements such as Walsh matrices [20]. Generally, it is preferable to use matrices of a known simple structure with integer and zero elements in order to avoid internal multiplications.

An important characteristic of the realizations based on the decomposition theorem is the possibility to obtain a multitude of them, depending on the choice of the matrices $\boldsymbol{R}$, $S$. Clearly, for example, the realizations suggested by (14) are not unique. Since each of these realizations is of the same general structure, we can choose in advance $\boldsymbol{R}$ and $S$ to achieve a desired objective, such as reduced roundoff noise, ease in the VLSI implementation, minimality, etc.

\section{Implications of the Decomposition Theorem AND REALIZABILITY}

In this section we consider the implications of the previous theorem to the design and realization of 2-D filters. Throughout this paper, the $z$-transform of a 2-D sequence $x(n, m)$, is defined by

$$
X\left(z_{1}, z_{2}\right)=\sum_{n=-\infty}^{\infty} \sum_{m=-\infty}^{\infty} x(n, m) z_{1}^{n} z_{2}^{m}
$$

where $z_{1}, z_{2}$ are delay elements [21].

The concept of feedback in 2-D filters is applied in a way similar to that of 1-D filters [22], [23].

In this section, a corollary of Theorem 1 relevant to the realization of 2-D filters is presented.

Corollary 1: The 2-D filter described by a rational transfer function of the form (1) can always be realized as a cascade of three blocks. A cascade block (C), an array block (A), and a feedback block (F) are defined in Fig. 3, in a manner shown in Fig. 4. 


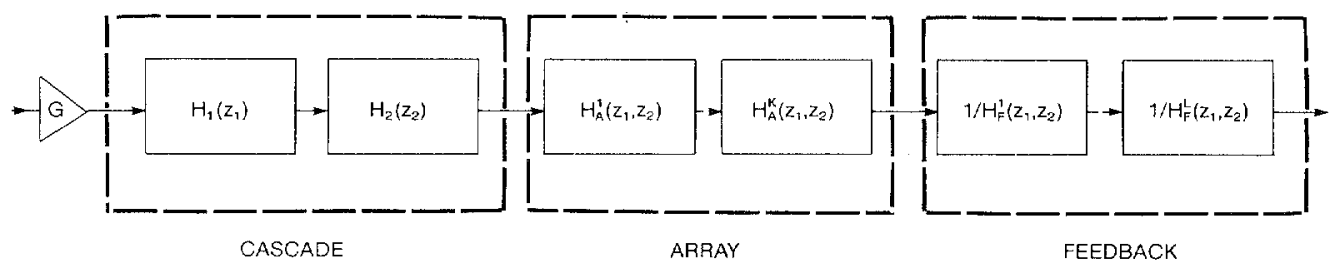

Fig. 3. Realization of general 2-D rational transfer function by the decomposition theorem.

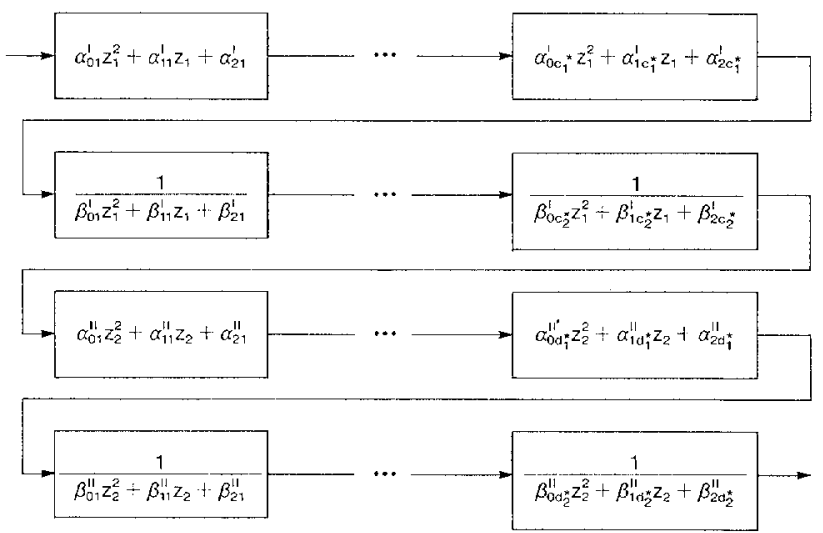

(a)

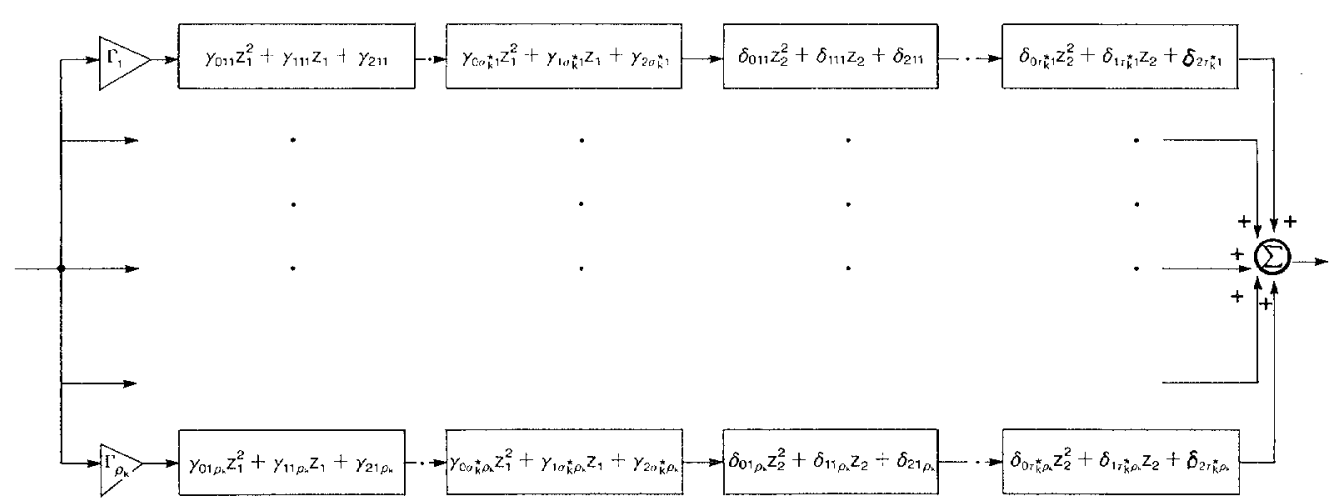

(b)

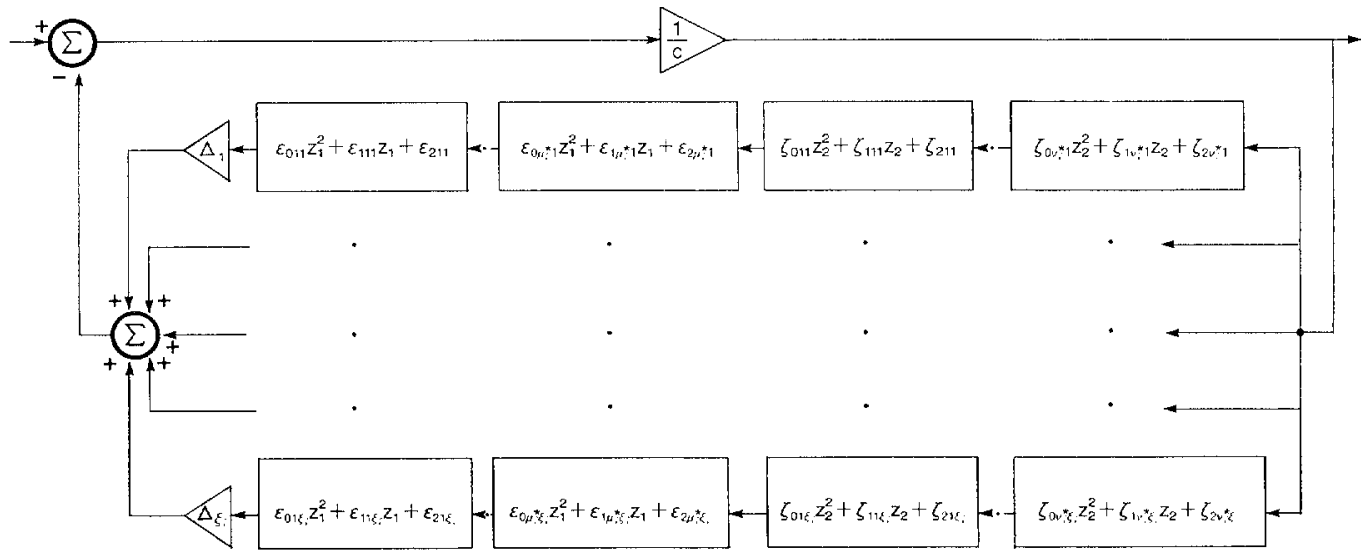

(c)

Fig. 4. (a). Realization of the cascade block of Fig. 3. (b). Realization of $H_{A}^{k}\left(z_{1}, z_{2}\right)$. (c). Realization of $1 / H_{F}^{\prime}\left(z_{1}, z_{2}\right)$. 

form

Proof: We can write the given transfer function in the

$$
\begin{aligned}
H\left(z_{1}, z_{2}\right) & =\frac{\sum_{i=0}^{n_{1}} \sum_{j=0}^{m_{1}} q_{i j} z_{1}^{i} z_{2}^{j}}{\sum_{i=0}^{n_{2}} \sum_{j=0}^{m_{2}} p_{i j} z_{1}^{i} z_{2}^{j}} \\
& =A H_{1}\left(z_{1}\right) H_{2}\left(z_{2}\right) H_{3}\left(z_{1}, z_{2}\right)
\end{aligned}
$$

where

$$
H_{3}\left(z_{1}, z_{2}\right)=\frac{H_{A}\left(z_{1}, z_{2}\right)}{H_{F}\left(z_{1}, z_{2}\right)}=\frac{\prod_{k=1}^{K} H_{A}^{k}\left(z_{1}, z_{2}\right)}{\prod_{l=1}^{L} H_{F}^{l}\left(z_{1}, z_{2}\right)}
$$

$H_{A}\left(z_{1}, z_{2}\right), H_{F}\left(z_{1}, z_{2}\right)$, are relatively prime polynomials, $H_{A}^{k}\left(z_{1}, z_{2}\right), H_{F}^{l}\left(z_{1}, z_{2}\right)$ are irreducible polynomials and $A$ is the constant gain.

The factors $H_{1}\left(z_{1}\right), H_{2}\left(z_{2}\right)$ can be realized by the cascade part [Fig. 4(a)], since they are functions of one variable. Similarly, the polynomial $H_{A}\left(z_{1}, z_{2}\right)$ and the rational function $1 / H_{F}\left(z_{1}, z_{2}\right)$ can be realized using $(8),(10)$ as shown in Fig. 4(b) and 4(c), respectively.

The following comments are in order:

1) (28) reveals that while a cascade of three general blocks (C, A, and F) is adequate to represent (1); it is also possible, and often desirable, to represent (1) as a cascade of more array and feedback terms, when $H_{A}\left(z_{1}, z_{2}\right)$ and $H_{F}\left(z_{1}, z_{2}\right)$ are reducible to lower order polynomials.

2) To avoid complex multiplications, first order complex conjugate terms can be combined, resulting in second order terms with real coefficients.

3) The forms of $H_{1}\left(z_{1}\right), H_{2}\left(z_{2}\right), H_{A}^{k}\left(z_{1}, z_{2}\right), H_{F}^{l}\left(z_{1}, z_{2}\right)$, are given by the formulas:

$$
\begin{aligned}
H_{1}\left(z_{1}\right)= & \frac{\sum_{i=0}^{c_{1}} q_{i}^{\mathrm{I}} z_{1}^{i}}{\sum_{i=0}^{c_{2}^{2}} p_{i}^{\mathrm{I}} z_{1}^{i}} \\
= & \sum_{i=1}^{c^{*}}\left[\alpha_{0 i}^{\mathrm{I}} z_{1}^{2}+\alpha_{1 i}^{\mathrm{I}} z_{1}+\alpha_{2 i}^{\mathrm{I}}\right] \sum_{i=1}^{c_{2}^{*}} \\
& \cdot\left[\frac{1}{\beta_{0 i}^{\mathrm{I}} z_{1}^{2}+\beta_{1 i}^{\mathrm{I}} z_{1}+\beta_{2 i}^{\mathrm{I}}}\right] \\
H_{2}\left(z_{2}\right)= & \frac{\sum_{i=0}^{d_{1}} q_{i}^{\mathrm{II}} z_{2}^{i}}{\sum_{i=0}^{d_{2}} p_{i}^{\mathrm{II}} z_{2}^{i}} \\
= & \sum_{i=1}^{d_{1}^{*}}\left[\alpha_{0 i}^{\mathrm{II}} z_{2}^{2}+\alpha_{1 i}^{\mathrm{II}} z_{2}+\alpha_{2 i}^{\mathrm{II}}\right] \sum_{i=1}^{d_{2}^{*}} \\
& \cdot\left[\frac{1}{\beta_{0 i}^{\mathrm{II}} z_{2}^{2}+\beta_{1 i}^{\mathrm{II}} z_{2}+\beta_{2 i}^{\mathrm{II}}}\right]
\end{aligned}
$$

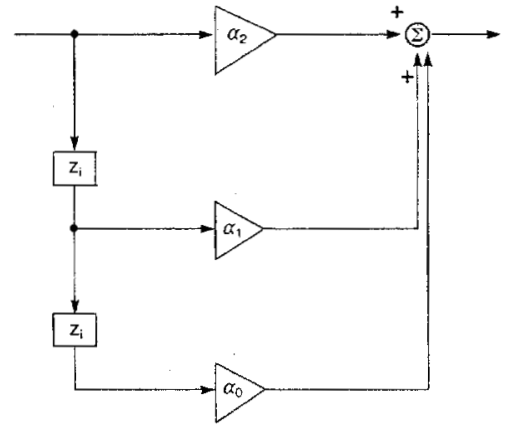

Fig. 5. Realization of $\alpha_{0} z_{1}^{2}+\alpha_{1} z_{1}+\alpha_{2}$.

$$
\begin{aligned}
& H_{A}^{k}\left(z_{1}, z_{2}\right)=\sum_{i=0}^{\sigma_{k}} \sum_{j=0}^{\tau_{j}} m_{i j}^{k} z_{1}^{i} z_{2}^{j} \\
& H_{F}^{l}\left(z_{1}, z_{2}\right)=\sum_{i=0}^{\mu l} \sum_{j=0}^{\nu_{l}} \eta_{i j}^{l} z_{1}^{i} z_{2}^{j}
\end{aligned}
$$

where $c_{1}^{*}, c_{2}^{*}, d_{1}^{*}, d_{2}^{*}, \sigma_{k}^{*}, \tau_{k}^{*}, \mu_{l}^{*}, \nu_{l}^{*}$ in Fig. 4 are the least integers equal or greater than $c_{1} / 2, c_{2} / 2, d_{1} / 2, d_{2} / 2, \sigma_{k} / 2$, $\tau_{k} / 2, \mu_{l} / 2, \nu_{l} / 2$, respectively.

Note that the following equations give the relation among the order $n_{1}, m_{1}, n_{2}, m_{2}$ of the given filter and the orders of factors $H_{1}\left(z_{1}\right), H_{2}\left(z_{2}\right), H_{A}^{k}\left(z_{1}, z_{2}\right), H_{F}^{l}\left(z_{1}, z_{2}\right)$

$$
\begin{array}{cc}
c_{1}+\sum_{k=1}^{K} \sigma_{k}=n_{1}, & d_{1}+\sum_{k=1}^{K} \tau_{k}=m_{1} \\
c_{2}+\sum_{l=1}^{L} \mu_{l}=n_{2}, & d_{2}+\sum_{l=1}^{L} \nu_{l}=m_{2} .
\end{array}
$$

Clearly, one of the factors $H_{1}\left(z_{1}\right), H_{2}\left(z_{2}\right)$ may be deleted (then $c_{1}=c_{2}=0$ or $d_{1}=d_{2}=0$ ) or may be all zero or all pole functions (then $c_{1}=0$ or $d_{1}=0$ and $c_{2}=0$ or $d_{2}$ $=0$, respectively).

The symbols $\rho_{k}$ and $\xi_{l}$ in Figs. 4(b) and 4(c) denote the number of parallel branches in the array and feedback blocks, respectively, and their values depend on the specific decomposition of the coefficient matrices of $H_{A}^{k}\left(z_{1}\right.$, $\left.z_{2}\right)$ and $H_{F}^{l}\left(z_{1}, z_{2}\right)$. As it has been previously explained

$$
\begin{aligned}
& \operatorname{rank} Q^{k} \leq \rho_{k} \leq \min \left(\sigma_{k}+1, \tau_{k}+1\right) \\
& \operatorname{rank} \boldsymbol{P}^{l} \leq \xi_{l} \leq \min \left(\mu_{l}+1, \nu_{l}+1\right)
\end{aligned}
$$

where $\boldsymbol{Q}^{k}, \boldsymbol{P}^{l}$ are the coefficient matrices of $H_{A}^{k}\left(z_{1}, z_{2}\right)$, $H_{F}^{l}\left(z_{1}, z_{2}\right)$, respectively.

4) One possible realization of the second order polynomial of the term

$$
\alpha_{0} z_{i}^{2}+\alpha_{1} z_{i}+\alpha_{2}
$$

is shown in Fig. 5 and that one possible realization of the term

$$
\frac{1}{\beta_{0} z_{i}^{2}+\beta_{1} z_{i}+\beta_{2}}
$$

is shown in Fig. 6, $i=1,2$.

A serious restriction in the implementation of the digital filters is realizability. Unrealizable delay-free loops must 


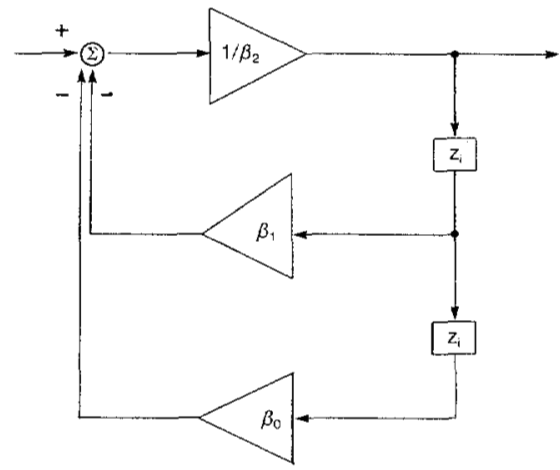

Fig. 6. Realization of $1 /\left(\beta_{0} z_{i}^{2}+\beta_{1} z_{i}+\beta_{2}\right)$.

be avoided [24] and this can be accomplished either by graph transformations of the resulting filter or by imposition of constraints on the decomposition. In the sequel, a theorem is presented describing a necessary and sufficient condition for realizability of filters obtained by the decomposition theorem.

Corollary 2: The existence of nonzero constant term in the denominator polynomial of (1) is a necessary and sufficient condition for the existence of implementations as in Fig. 2 without delay free-loops, which are functions of positive powers of $z_{1}$ and $z_{2}$.

The above corollary can be easily verified by the following proof.

Proof: Let the denominator polynomial be

$$
p\left(z_{1}, z_{2}\right)=c+p_{1}\left(z_{1}, z_{2}\right), \quad c \neq 0 .
$$

Then the polynomial $p_{1}\left(z_{1}, z_{2}\right)$ can be written in the form of (2), where the corresponding matrix $\boldsymbol{P}_{1}$ is of the form

$$
\boldsymbol{P}_{1}=\left[\begin{array}{cccc}
0 & p_{01} & \cdots & p_{0 m_{1}} \\
p_{10} & p_{11} & \cdots & p_{1 m_{1}} \\
\vdots & \vdots & & \vdots \\
p_{n_{1} 0} & p_{m_{1} 1} & \cdots & p_{n_{1} m_{1}}
\end{array}\right] .
$$

Consider the decomposition given by (14), where the auxiliary matrix $\boldsymbol{R}$ is taken into account. Let this matrix $\boldsymbol{R}$ be a nonsingular matrix of the following form

$$
\boldsymbol{R}=\left[\begin{array}{cccc}
r_{00} & 0 & \cdots & 0 \\
r_{10} & r_{11} & \cdots & r_{1 n 1} \\
\vdots & \vdots & & \vdots \\
r_{n 10} & r_{n 11} & \cdots & r_{n|n|}
\end{array}\right]
$$

For

$$
\begin{aligned}
r_{0 i} & \neq 0, & i & =0 \\
& =0, & i & =1,2, \cdots, n_{1} .
\end{aligned}
$$

We can easily show that the polynomials $r_{i}\left(z_{1}\right), i=1,2$, $\cdots, n_{1}$ and $s_{0}\left(z_{2}\right)$, in the counterpart of $(8)$ for $p\left(z_{1}, z_{2}\right)$, do not have a constant term. Therefore, the counterpart of (8) for $p\left(z_{1}, z_{2}\right)$ does not contain a constant term, and the realization will not contain delay-free loops. If we use in the decomposition given by (14), a nonsingular matrix $S$, then the elements $s_{i 0}, i=1, \cdots, m_{1}$ of the matrix $S$ are chosen to be zero and the resulting realization does not contain delay-free loops.

\section{The Reconfigurable Filter}

Since Theorem 1 gives a general decomposition for a 2D rational transfer function, it can be used for the construction of a general reconfigurable filter, which can realize all rational transfer functions up to a given order. Corollary 2 is an obvious consequence of Theorem 1 and describes such a filter.

Corollary 3: Let the given transfer function be of the form (1). Then setting

$$
\begin{aligned}
c_{1} & =n_{1}, & c_{2} & =n_{2} \\
d_{1} & =m_{1}, & d_{2} & =m_{2} \\
\sigma & =\sum_{k=1}^{K} \sigma_{k}=n_{1}, & \tau & =\sum_{k=1}^{K} \tau_{k}=m_{1} \\
\mu & =\sum_{l=1}^{L} \mu_{l}=n_{2}, & \nu & =\sum_{l=1}^{L} \nu_{l}=m_{2}
\end{aligned}
$$

in the configuration of Fig. 4, all the possible transfer functions (1) up to a given order can be realized, with proper specification of the following parameters:

$G$,

$$
\begin{aligned}
& \alpha_{0 i}^{\mathrm{I}}, \quad \alpha_{1 i}^{\mathrm{I}}, \quad \alpha_{2 i}^{\mathrm{I}}, \quad i=1, \cdots, c_{1}^{*} \\
& \alpha_{0 i}^{\mathrm{II}}, \quad \alpha_{1 i}^{\mathrm{II}} \quad \alpha_{2 i}^{\mathrm{II}}, \quad i=1, \cdots, c_{2}^{*} \\
& \beta_{0 i}^{\mathrm{I}}, \quad \beta_{1 i}^{\mathrm{I}} \quad \beta_{2 i}^{\mathrm{I}}, \quad i=1, \cdots, d_{1}^{*} \\
& \beta_{0 i}^{\mathrm{II}}, \quad \beta_{1 i}^{\mathrm{II}} \quad \beta_{2 i}^{\mathrm{II}}, \quad i=1, \cdots, d_{2}^{*} \\
& \gamma_{0 i j}, \quad \gamma_{1 i j} \quad \gamma_{2 i j}, \quad i=1, \cdots, \sigma_{k}^{*}, \\
& j=1, \cdots, \rho_{k} \\
& \left.\delta_{0 i j}, \quad \delta_{1 i j} \quad \delta_{2 i j}, \quad i=1, \cdots, \tau_{k}^{*}, \quad\right\} k=1, \cdots, K \\
& j=1, \cdots, \rho_{k} \\
& \epsilon_{0 i j}, \quad \epsilon_{1 i j} \quad \epsilon_{2 i j}, \quad i=1, \cdots, \mu_{l}^{*} \\
& \left.\begin{array}{rl}
j & =1, \cdots, \xi_{l} \\
\zeta_{0 i j}, \quad \zeta_{1 i j} \quad \zeta_{2 i j}, \quad i & =1, \cdots, \nu_{l}^{*}, \\
j & =1, \cdots \xi_{l}
\end{array}\right\} l=1, \cdots, L \\
& \Gamma_{i}, \quad i=1, \cdots, \rho_{k}, \quad k=1, \cdots, K \\
& \Delta_{i}, \quad i=1, \cdots, \xi_{l}, \quad l=1, \cdots, L .
\end{aligned}
$$

Note that for convenience, without loss of generality, the coefficients of the second order terms $z_{1}^{2}, z_{2}^{2}$ (i.e., $\alpha_{0 i}^{1}$, $\left.\alpha_{0 i}^{\mathrm{II}}, \beta_{0 i}^{\mathrm{I}}, \beta_{0 i}^{\mathrm{II}}, \gamma_{0 i j}, \delta_{0 i j}, \epsilon_{0 i j}, \zeta_{0 i j}\right)$ take only the values zero or one. 
The proof is straightforward and is omitted.

Note that by adjusting the above parameters, any filter up to a given order can be realized. Corollary 2 is of interest because it leads to a system with a highly modular structure and parallelism, which can be used to satisfy different requirements [25].

Also note that the first order terms resulting from Theorem 1 can also be combined in other ways to provide other desirable modular expansions.

We finally propose the basic steps of an algorithm for the implementation of 2-D filters, as follows:

1) Given a 2-D rational function $H\left(z_{1}, z_{2}\right)$, use the primitive factorization method [6] which allows the extraction of 1-D polynomials $H_{1}\left(z_{1}\right), H_{2}\left(z_{2}\right)$.

2) Remove the common factors of the numerator and denominator left [7], [8].

3) If the resulting numerator and denominator are not irreducible, expand them in terms of lower order polynomials in $z_{1}, z_{2}$ [7], [8]. Now the given transfer function can be written in the form of (27) and (28).

4) Realize the cascade terms $H_{1}\left(z_{1}\right), H_{2}\left(z_{2}\right)$, by any of the well known 1-D filter realization methods (cascade, array, canonic, etc.).

5) Consider the remaining function $H_{A}\left(z_{1}, z_{2}\right) / H_{F}\left(z_{1}\right.$, $z_{2}$ ), which does not contain 1-D polynomials in the numerator or denominator. Realize each irreducible factor $H_{A}^{k}\left(z_{1}, z_{2}\right), k=1, \cdots, K$ of $H_{A}\left(z_{1}, z_{2}\right)$, by the array block of the form given in Fig. 4(b) and each irreducible factor $1 / H_{F}^{l}\left(z_{1}, z_{2}\right), l=1, \cdots, L$ of $1 / H_{F}\left(z_{1}, z_{2}\right)$, by the cascade block of the form given in Fig. 4(c). Examples follow:

\section{Example 1:}

Consider the low-pass 2-D digital filter obtained by Maria and Fahmy [26]:

Its magnitude response is specified by

$$
Y_{m n} \begin{cases}=1 & \left(w_{1}^{2}+w_{2}^{2}\right)^{1 / 2} \leq 0.08 \\ =0.5 & 0.08<\left(w_{1}^{2}+w_{2}^{2}\right)^{1 / 2}<0.12 \\ =0 & \left(w_{1}^{2}+w_{2}^{2}\right)^{1 / 2} \geq 0.12 .\end{cases}
$$

Its transfer function designed by a computer-aided design technique [26] was
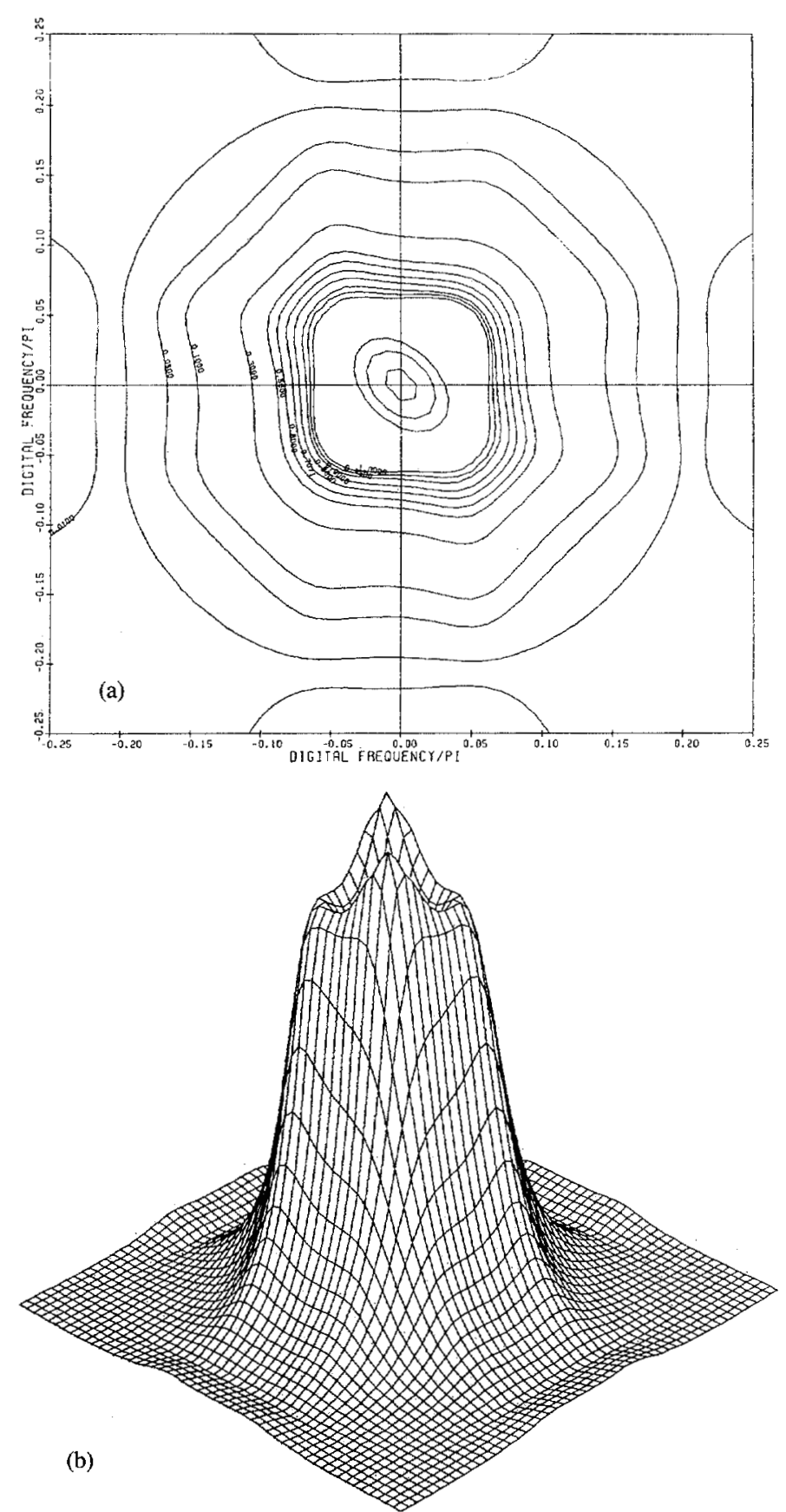

Fig. 7. (a). Contour plot of magnitude response of low-pass filter $\mathrm{H}_{2}\left(z_{1}\right.$, $z_{2}$ ). (b). Perspective plot of magnitude response of low-pass filter $H_{2}\left(z_{1}\right.$, $\left.z_{2}\right)$.

$$
H_{1}\left(z_{1}, z_{2}\right)=(0.00895) \frac{\left[\begin{array}{lll}
1 & z_{1} & z_{1}^{2}
\end{array}\right]\left[\begin{array}{ccc}
1 & -1.62151 & 0.99994 \\
-1.62151 & 2.63704 & -1.621299 \\
0.99994 & -1.62129 & 1.00203
\end{array}\right]\left[\begin{array}{l}
1 \\
z_{2} \\
z_{2}^{2}
\end{array}\right]}{\left[\begin{array}{lll}
1 & z_{1} & z_{1}^{2}
\end{array}\right]\left[\begin{array}{rrr}
-1.78813 & 3.20640 & -1.49271 \\
0.82930 & -1.49271 & 0.69823
\end{array}\right]\left[\begin{array}{l}
1 \\
z_{2} \\
z_{2}^{2}
\end{array}\right]}
$$

Its magnitude response is shown in Fig. 7(a) and (b). 
TABLE I

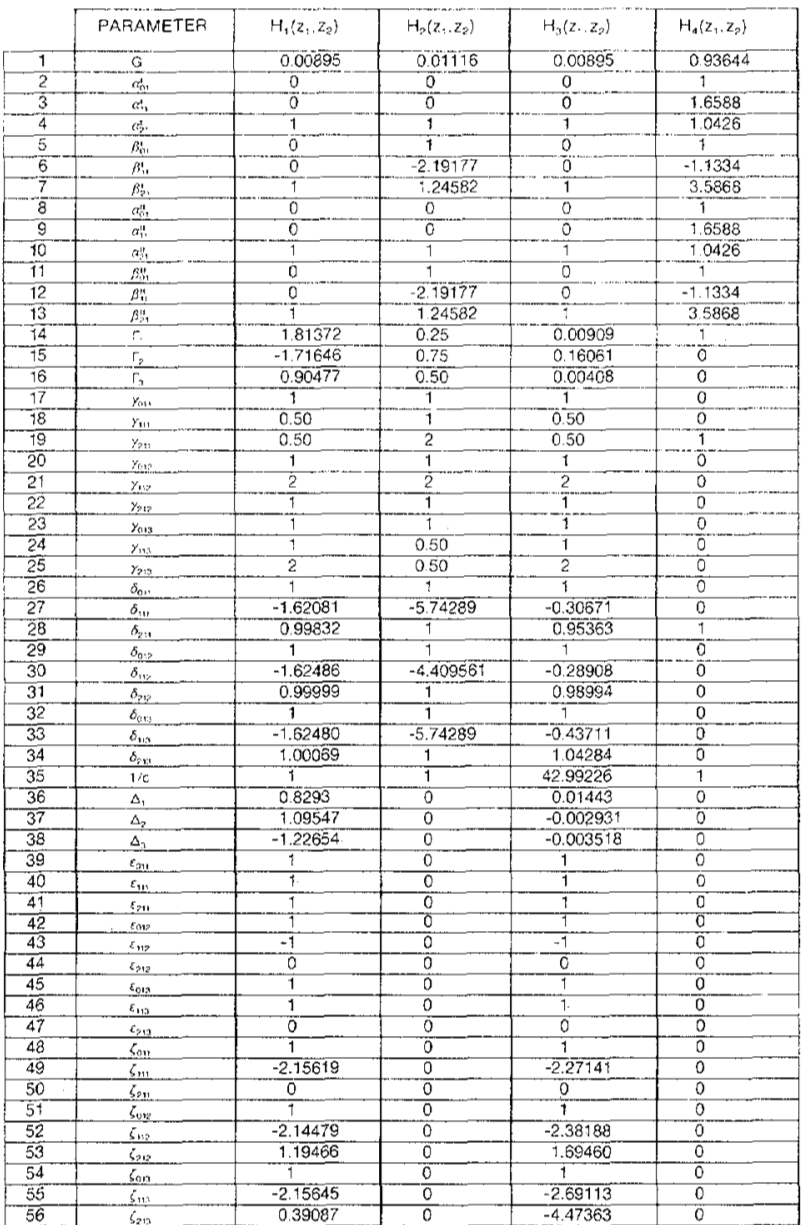

After carrying out the steps of the proposed algorithm, with

$$
\boldsymbol{R}=\left[\begin{array}{lll}
1 & 1 & 2 \\
1 & 2 & 1 \\
2 & 1 & 1
\end{array}\right]
$$

for the numerator and

$$
\boldsymbol{R}=\left[\begin{array}{rrr}
1 & 0 & 0 \\
1 & 1 & 1 \\
1 & -1 & 1
\end{array}\right]
$$

for the denominator, we arrive at the realization specified in Table I in terms of the parameters of Fig. 4(a), (b), and (c).

A filter with the same specifications was designed by using the technique of [27]. This technique makes use of symmetries in the magnitude response and results in a simpler optimization and a filter with a separable denominator. The resulting transfer function was

$$
\begin{aligned}
H_{2}\left(z_{1}, z_{2}\right)= & 7.190551 \times 10^{-3} \\
{\left[\begin{array}{lll}
1 & z_{1} & z_{2}^{2}
\end{array}\right]\left[\begin{array}{ccc}
0 & 1 & 0 \\
1 & -3.742894 & 1 \\
0 & 1 & 0
\end{array}\right]\left[\begin{array}{l}
1 \\
z_{2} \\
z_{2}^{2}
\end{array}\right] } & \times \frac{\left(1-1.759295 z_{1}+0.8026835 z_{1}^{2}\right)\left(1-1.759295 z_{2}+0.8026835 z_{2}^{2}\right)}{(1-10} .
\end{aligned}
$$
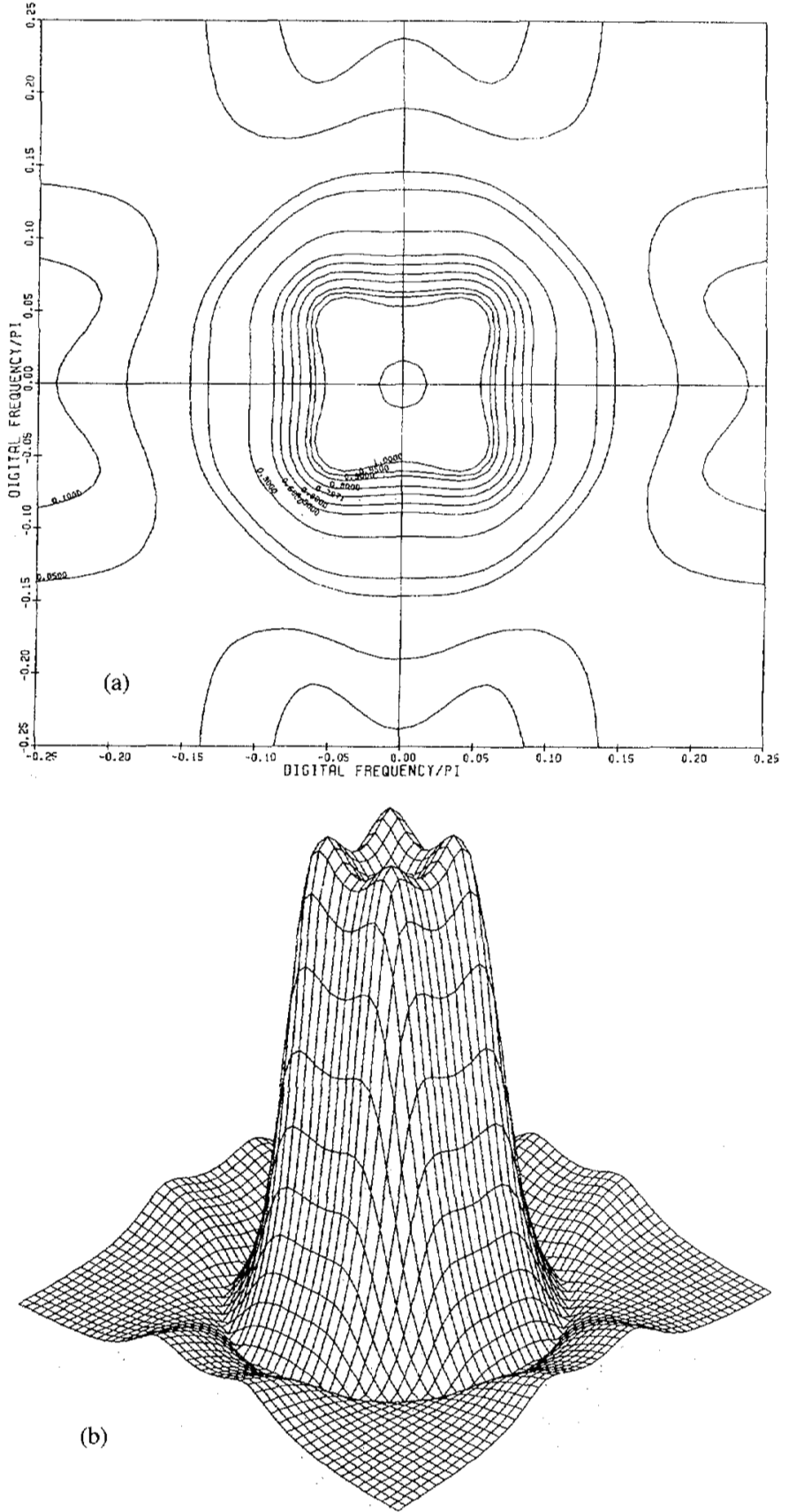

Fig. 8. (a). Contour plot of magnitude response of low-pass filter $H_{1}\left(z_{1}\right.$, $\left.z_{2}\right)$. (b). Perspective plot of magnitude response of low-pass filter $H_{1}\left(z_{1}\right.$, $\left.z_{2}\right)$.

The magnitude response for the second filter is shown in Fig. 8(a) and (b). Using

$$
\boldsymbol{R}=\left[\begin{array}{lll}
2 & 1 & 1 \\
1 & 2 & 1 \\
1 & 1 & 2
\end{array}\right]
$$




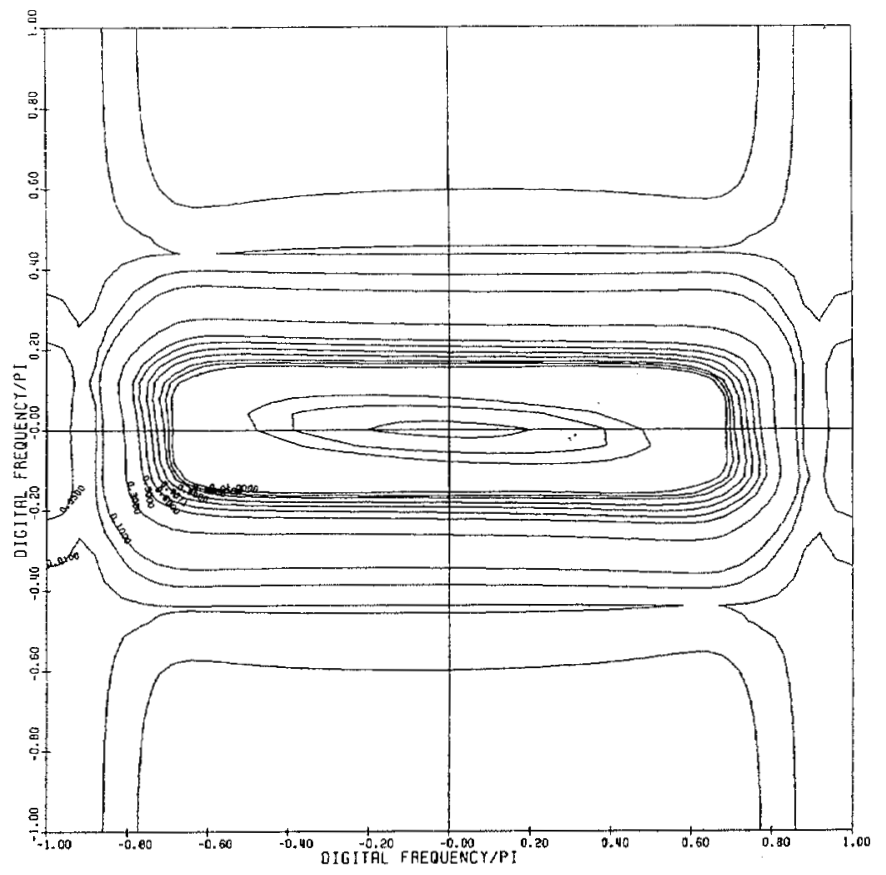

(a)

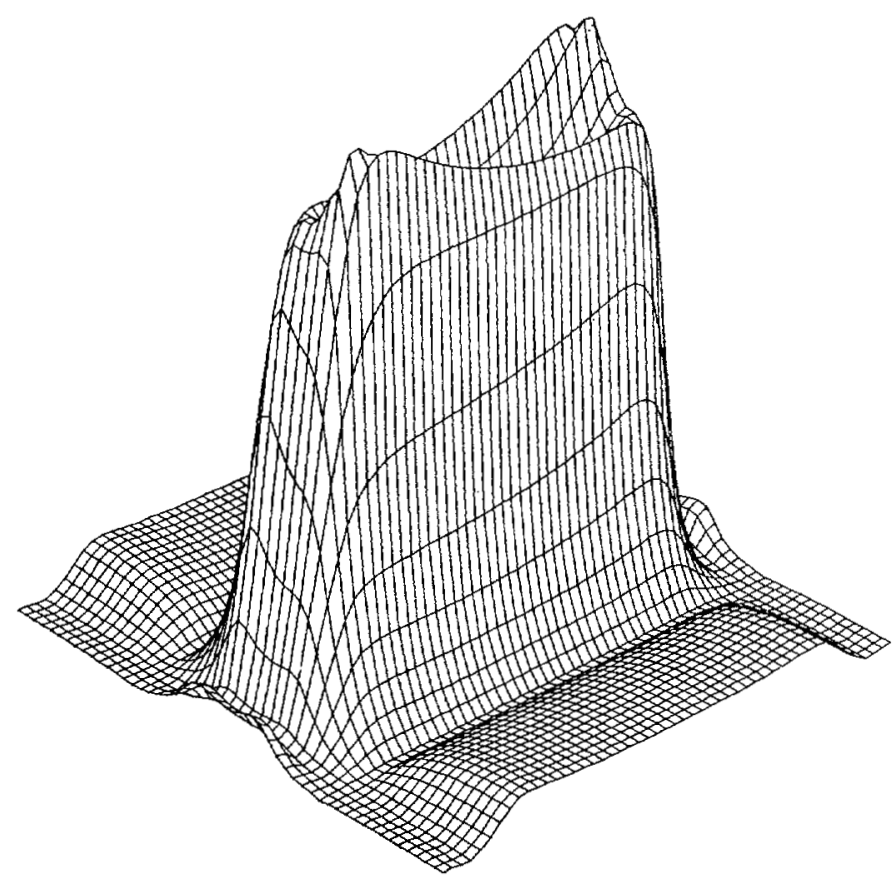

(b)

Fig. 9. (a). Contour plot of magnitude response of transformed filter $H_{3}\left(z_{1}\right.$, $z_{2}$ ). (b). Perspective plot of magnitude response of transformed filter $H_{3}\left(z_{1}, z_{2}\right)$.

for the numerator, the realization given in Table I was obtained. The denominator was already in cascade form.

Using the spectral transformation technique of [28] on $H_{1}\left(z_{1}, z_{2}\right)$, the filter becomes can be considered as special cases of the filter described in Section IV, where $c=e=\sigma=\mu=d=f=\tau=\nu=$ 2 and some parameters of the general configuration are set equal to zero or one in each case.

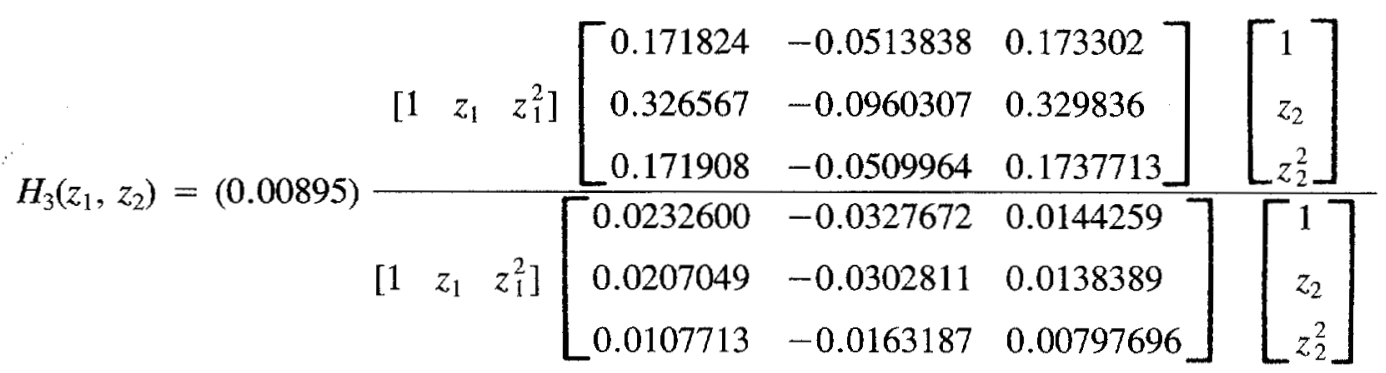

whose magnitude response is shown in Fig. 9(a) and (b).

Carrying out the steps of the algorithm, with the same $\boldsymbol{R}$ for the numerator and the denominator, as in the case of $H_{1}\left(z_{1}, z_{2}\right)$, we arrive at the realization given in Table I.

We finally consider the low-pass filter given in [29], with a separable transfer function

$$
H_{4}\left(z_{1}, z_{2}\right)=H\left(z_{1}\right) H\left(z_{2}\right)
$$

where

$$
\begin{aligned}
H(z) & =0.2813 \frac{1+1.591 z+0.9591 z^{2}}{1-0.3161+0.27788 z^{2}} \\
& =0.9677 \frac{z^{2}+1.6588 z+1.0426}{z^{2}-1.1334 z+3.5868} .
\end{aligned}
$$

The magnitude response of $H_{4}\left(z_{1}, z_{2}\right)$ is shown in Fig. 10(a) and 10(b) and its realization in Table I.

Clearly, the configurations of the previous four filters
V. Stability Considerations of Decomposed Filters

Since the decomposition theorem deals with the general realization of 2-D filters, IIR filters that were checked for stability, by any of the well known stability theorems [30][36] will result in stable realizations.

The various known stability conditions expressed either on the transfer function or the impulse response, contain the parameters (coefficients) of the filter, so that BIBO stability is satisfied. Other equivalent conditions can be obtained by applying known stability conditions to modular realizations. These constrains, expressed over the new parameters of the filter, are generally quite involved.

However, the existence of a large choice of modular realizations indicates that it may be worthwhile to search for those realizations, which result in stability conditions that can be expressed in a simple way in terms of the parameters involved. Similar approaches can be used in the search for appropriate stabilization techniques. 


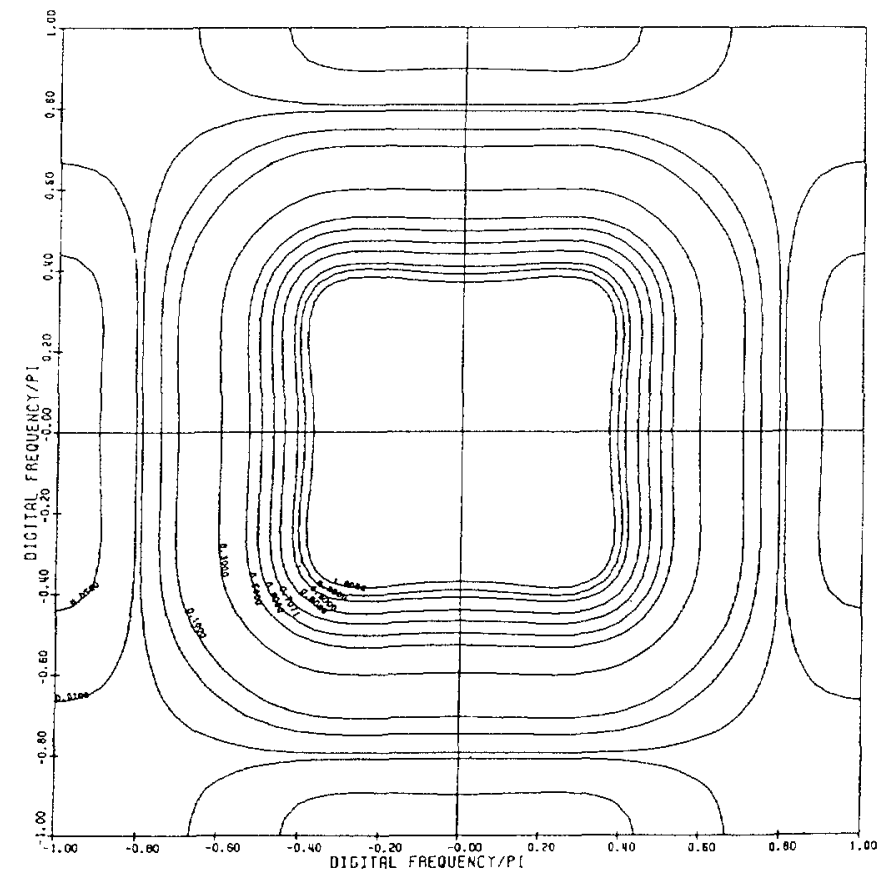

(a)

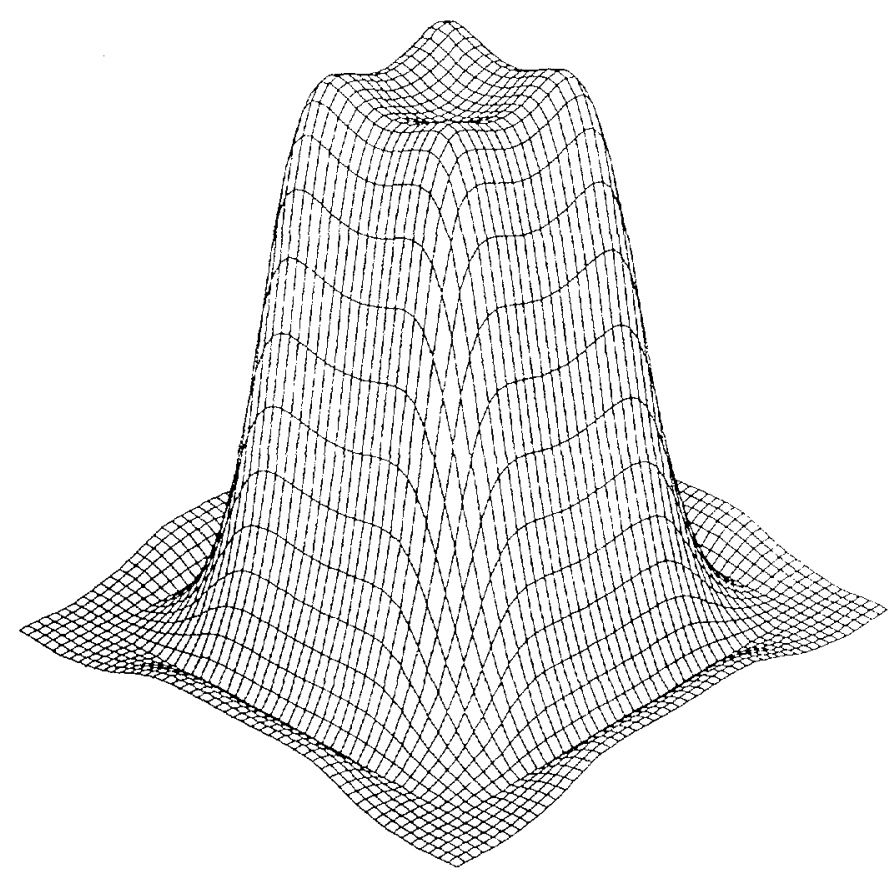

(b)

Fig. 10. (a). Contour plot of magnitude response of low-pass separable filter $H_{4}\left(z_{1}, z_{2}\right)$. (b). Perspective plot of magnitude response of low-pass separable filter $H_{4}\left(z_{1}, z_{2}\right)$.

In the remainder of this section we outline the approach proposed.

Consider the well known condition for stability of a causal 2-D filter, expressed in terms of the unit pulse response [21], [30]

$$
\sum_{n=-\infty}^{\infty} \sum_{m=-\infty}^{\infty}|h(n, m)|<\infty .
$$

Note that the unit pulse response $h(n, m)$ is related to the transfer function of the filter by the relationship

$$
H\left(z_{1}, z_{2}\right)=\sum_{n=-\infty}^{\infty} \sum_{m=-\infty}^{\infty} h(n, m) z_{1}^{n} z_{2}^{m} .
$$

Usually, stability depends only on the denominator of the transfer function (1) (excluding the case where nonessential singularities of second kind exist [36]). Here, we only examine filters having a constant numerator.

Consider now a fixed matrix $\boldsymbol{R}$ and use (3) to write the unit pulse response $h(n, m)$ as follows

$$
\begin{aligned}
h(n, m) & =Z^{-1}\left[\frac{1}{p\left(z_{1}, z_{2}\right)}\right] \\
& =Z^{-1}\left[\left[1+Z_{1}^{T} \boldsymbol{R S Z}_{2}\right]^{-1}\right]
\end{aligned}
$$

where $Z^{-1}$ denotes the 2-D inverse $z$ transform and $S=$ $\boldsymbol{R}^{-1} \cdot \boldsymbol{P}_{1}$, where $\boldsymbol{P}_{1}$ is the matrix of the polynomial $p_{1}\left(z_{1}\right.$, $\left.z_{2}\right)$.

(37) substituted in (36) results in a stability condition expressed in terms of the parameters of the decomposition, which depend on the choice of $\boldsymbol{R}$ and the region of convergence of $H\left(z_{1}, z_{2}\right)$. Different choices of $\boldsymbol{R}$ will result in different stability conditions. The evaluation of (36), (37) does not lead in general to an easy stability test. They are the conditions which have to be satisfied in order to ensure stability.

In the particular case of image processing, where both variables of the unit pulse response $h(n, m)$ are space variables, causality constraints do not exist. In this case, it is possible to guarantee stability by selecting the order of recursion of the component filters of the modular realization. In practical applications which deal with finite extent images, adequate scaling should be applied to guarantee no overflow of the output.

\section{Example 2:}

Consider the filter with the transfer function of the form

$$
H\left(z_{1}, z_{2}\right)=\frac{1}{1+a z_{1}+b r_{2}+c z_{1} z_{2}}=\frac{1}{1+p_{1}\left(z_{1}, z_{2}\right)}
$$

where $p_{1}\left(z_{1}, z_{2}\right)=a z_{1}+b z_{2}+c z_{1} z_{2}$. The corresponding matrix $P_{1}$ is

$$
\boldsymbol{P}_{1}=\left[\begin{array}{ll}
0 & b \\
a & c
\end{array}\right]
$$

Here, we have three independent nonzero parameters, $a$, $b$, and $c$. We shall consider the realization using the Jordan form. To this end let $\lambda_{1}, \lambda_{2}$ be the eigenvalues of $\boldsymbol{P}_{1}$. Then it can be found easily that

$$
\boldsymbol{H}=\left[\begin{array}{ll}
1 & 1 \\
\lambda_{1} / b & \lambda_{2} / b
\end{array}\right]
$$

and

$$
\boldsymbol{H}^{-1}=\left[\begin{array}{rr}
\lambda_{2} / b & -1 \\
-\lambda_{1} / b & 1
\end{array}\right] \frac{b}{\lambda_{2}-\lambda_{1}} .
$$




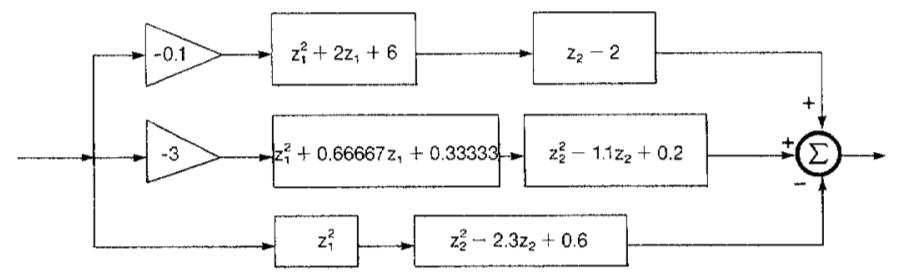

Fig. 11. Realization of the numerator of the filter of Example 2.

Then $p 1\left(z_{1}, z_{2}\right)$ can be written as

$$
\begin{aligned}
p_{1}\left(z_{1}, z_{2}\right)= & \frac{b}{\lambda_{2}-\lambda_{1}}\left[\begin{array}{ll}
1 & z_{1}
\end{array}\right]\left[\begin{array}{cc}
1 & 1 \\
\lambda_{1} / b & \lambda_{2} / b
\end{array}\right]\left[\begin{array}{cc}
\lambda_{1} & 0 \\
0 & \lambda_{2}
\end{array}\right] \\
& \cdot\left[\begin{array}{rr}
\lambda_{2} / b & -1 \\
-\lambda_{1} / b & 1
\end{array}\right]\left[\begin{array}{c}
1 \\
z_{2}
\end{array}\right] \\
= & \boldsymbol{Z}_{1}^{T} \boldsymbol{P}_{1} Z_{2}
\end{aligned}
$$

where

$$
\hat{\boldsymbol{P}}_{1}=\left[\begin{array}{cc}
0 & b \\
-\lambda_{1} \lambda_{2} / b & \lambda_{1}+\lambda_{2}
\end{array}\right] \text {. }
$$

The polynomial $p_{1}\left(z_{1}, z_{2}\right)$ is written in the form of $(3)$, with the matrix $R$, in the form of (33), in order to obtain a realizable implementation. In particular, by choosing

$$
\boldsymbol{R}=\left[\begin{array}{ll}
1 & 0 \\
1 & 1
\end{array}\right]
$$

we obtain the decomposed form of Fig. 12 .

The relationships between $a, b, c$ and the eigenvalues can be easily determined by equating the decomposed structure to the original one. Thus, we obtain

$$
\begin{aligned}
a b & =-\lambda_{1} \lambda_{2} \\
c & =\lambda_{1}+\lambda_{2} .
\end{aligned}
$$

In the proposed realization we must also have three independent parameters. To this end we consider as the third parameter the element $\lambda_{1} / b=x$ of the matrix $H$, in addition to the two eigenvalues ${ }^{1}$ (Fig. 12).

It was shown in [37], [38] that the necessary and sufficient conditions for the filter $H\left(z_{1}, z_{2}\right)$ to be stable in the ++ direction, i.e., for $1+a z_{1}+b z_{2}+c z_{1} z_{2} \neq 0 \vee\left(z_{1}\right.$, $\left.z_{2}\right) \in \bar{U}^{2} \triangleq\left\{\left(z_{1}, z_{2}\right):\left|z_{1}\right| \leq 1,\left|z_{2}\right| \leq 1\right\}$, are

$$
\begin{array}{r}
a+b-c<1 \\
a-b+c<1 \\
-a+b+c<1 \\
-a-b-c<1 .
\end{array}
$$

\footnotetext{
${ }^{1}$ Since $\lambda_{1}$ is already a parameter in the new representation, we could consider that $b$ remains the third parameter.
}

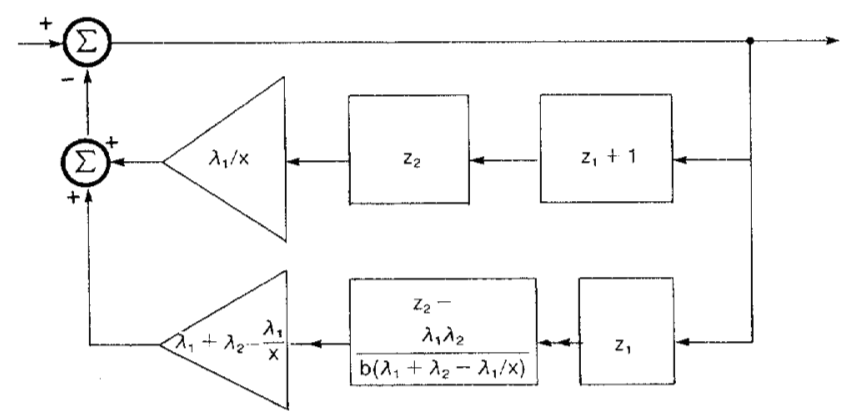

Fig. 12. Realization of the denominator of the filter of Example 2.

Since there is equivalence between the two expressions of the polynomial $p_{1}\left(z_{1}, z_{2}\right)$, we can express the above stability conditions in terms of the new independent parameters $\lambda_{1}, \lambda_{2}, x$. After some algebraic manipulations we obtain

$$
\begin{aligned}
& \lambda_{2} x^{2}+\left(\lambda_{1}+\lambda_{2}+1\right) x-\lambda_{1}>0 \\
& \lambda_{2} x^{2}-\left(\lambda_{1}+\lambda_{2}-1\right) x+\lambda_{1}>0 \\
& \lambda_{2} x^{2}+\left(\lambda_{1}+\lambda_{2}-1\right) x+\lambda_{1}<0 \\
& \lambda_{2} x^{2}-\left(\lambda_{1}+\lambda_{2}+1\right) x-\lambda_{1}<0
\end{aligned} \quad x>0
$$

and

$$
\begin{aligned}
& \lambda_{2} x^{2}+\left(\lambda_{1}+\lambda_{2}+1\right) x-\lambda_{1}<0 \\
& \lambda_{2} x^{2}-\left(\lambda_{1}+\lambda_{2}-1\right) x+\lambda_{1}<0 \\
& \lambda_{2} x^{2}+\left(\lambda_{1}+\lambda_{2}-1\right) x+\lambda_{1}>0 \\
& \lambda_{2} x^{2}-\left(\lambda_{1}+\lambda_{2}+1\right) x-\lambda_{1}>0
\end{aligned} \quad x<0
$$

Considering the following filter given in [37]

$$
H\left(z_{1}, z_{2}\right)=\frac{1}{1+0.5 z_{1}+0.01 z_{2}+0.4 z_{1} z_{2}} .
$$

Checking both sets of parameters, we conclude that the filter is stable. In the case that the filter was unstable, we could consider other orders of recursion, which correspond to other stability conditions.

\section{Conclusions and Possible Extensions}

Since it is possible to expand any rational function of a given order in two variables, in terms of first order functions of one variable, great modularity can be achieved in 2-D filter realizations. The degrees of freedom allowed by the choice of the matrices can be utilized for optimum filter implementation. This means that the coefficients in the decomposed form, which are influenced by the choice of the auxiliary matrices, can be properly determined to optimize some performance index. Thus, optimal filters can be designed, in the sense of this performance index.

The general structure described in the corollaries can be utilized in conjunction with computer-aided design techniques, for the optimum choice of the filter parameters. The generality of this method leads to adaptive and 
reconfigurable filters. Stability of the filters depends on the parameters of (1) and different possible realizations do not affect it. However, since the choice of parameters leading to a given realization is related to the filter coefficients, it is possible to obtain conditions that these parameters should satisfy to guarantee stability. Different filter structures lead to different noise properties when implemented by finite precision arithmetic [39]. Such considerations, the extension of the previous results to $M$-dimensions, and the appropriate choice of matrices $\boldsymbol{R}$ or $S$ different kinds of implementations, are presently under way.

\section{REFERENCES}

[1] T. S. Huang, W. F. Schreiber, and O. J. Tretiak, "Image processing," Proc. IEEE, vol. 59, pp. 1586-1609, 1971.

[2] G. A. Biiss, "Algebraic functions," New York Amer. Math. Soc., 1933.

[3] S. Chakrabarti, N. K. Bose, and S. K. Mitra, "Sum and product separabilities of multivariable functions and applications," J. Franklin Inst., vol. 299, pp. 53-66, 1975.

[4] S. Chakrabarti and S. K. Mitra, "An algorithm for multivariable polynomial factorization," Proc. IEEE Int. Symp. Circuits Syst., pp. $678-683,1977$.

[5] E. W. Kamen and R. M. Mersereau, "Exact and approximate decomposability of two-dimensional transfer functions," USARO Tech. Rep., 1976.

[6] M. Morf, B. C. Levy, and S.-Y. Kung, "2-D polynomial matrices, factorization and coprimeness," Proc. IEEE, vol. 65, pp. 861-872, 1977.

[7] N. K. Bose, "A criterion to determine if two multivariable polynomials are relatively prime," Proc. IEEE, vol. 60, pp. 134-135, 1972.

[8] — " "An algorithm for GCF extraction from two multivariable polynomials," Proc. IEEE, vol. 64, pp. 185-186, 1976

[9] R. Eising, "Separability of 2-D transfer matrices," IEEE Trans. Auto. Contr., vol. AC-24, pp. 508-510, 1979.

[10] P. N. Paraskevopoulos and B. G. Mertzios, "Factorization of SISO 2D transfer function," Int. J. Syst. Sci., vol. 12, pp. 1135-1147, 1981.

[11] S. Treitel and J. L. Shanks, "The design of multistage separable planar filters," IEEE Trans. Geosci., Electron., vol. GE-9, pp. 10 27, 1971.

[12] P. Pistor, "Stability criterion for recursive filters," IBM J. Res. Dev., vol. 18, pp. 59-71, 1974.

[13] M. P. Ekstrom and J. W. Woods, "Two-dimensional spectral factorization with applications in recursive digital filtering," IEEE Trans. Acoust., Speech, Signal Processing, vol. ASSP-24, pp. 115-128, 1976.

[14] A. Peled and B. Liu, "A new hardware realization of digital filters," IEEE Trans. Acoust., Speech, Signal Processing, vol. ASSP-22, pp. $456-462,1974$.

[15] A. N. Venetsanopoulos and B. G. Mertzios, "A general implementation technique for 2-D digital filters," Proc. 6th Summer Symposium Circuit Theory, Prague, Czechoslovakia, pp. 176-180, July 12-16, 1982.

[16] H. C. Andres and C. L. Patterson, "Singular value decompositions and digital image processing," IEEE Trans. Acoust., Speech, Signal Processing, vol. ASSP-24, pp. 26-53, Feb. 1976.

[17] P. Karivaratharajan and M. N. S. Swamy, "Realization of a 2-dimensional FIR digital filter using separable filters," Electron. Lett., vol. 14, pp. 249-251, Apr. 1978.

[18] A. N. Venetsanopoulos and C. L. Nikias, "Realization of two-dimensional digital filters by LU decomposition of their transfer function," in Proc. IEEE Int. Conf. Acoust., Speech, Signal Processing, San Diego, CA, pp. 20.4.1-20.4.4, Mar. 1984.

[19] A. P. Chrysafis, C. L. Nikias, and A. N. Venetsanopoulos, "Design and realization of two-dimensional FIR digital filters by LU decomposition of their impulse response," in Proc. IEEE Int. Symp. Circuits Systems, Montreal, pp. 1234-1037, May 1984.

[20] B. G. Mertzios and A. N. Venetsanopoulos, "Walsh matrix implementation of M-D filters by the decomposition theorem," in Proc. Int. Conf. Digital Signal Processing, Florence, Italy, pp. 64-68, Sept. 1984.

[21] G. Garibotto, "Two-dimensional recursive digital filters in picture processing," CSELT Rapporti Technici, vol. V, no. 1, Mar. 1977.

[22] B. G. Mertzios and P. N. Paraskevopoulos, "On the input-output de- coupling of 2-D systems by state feedback," J. Franklin Inst., vol. 314 , pp. $55-76,1982$.

[23] D. E. Dudgeon, "An iterative implementation for 2-D digital filters," IEEE Trans. Acoust., Speech, Signal Processing, vol. ASSP-28, pp. $666-671,1980$.

[24] L. T. Bruton, "Low-sensitivity digital ladder filters," IEEE Trans. Circuits Syst., vol. CAS-22, no. 3, p. 171, 1975.

[25] W. L. Kelly, H. F. Benz, and B. D. Meredith, "Needs: Information adaptive system," AIAA Sens. Syst. 80's Conf., Colorado Springs, CO, paper 80-1906, pp. 1-6, Dec. 2-4, 1980.

[26] G. A. Maria and M. M. Fahmy, "An $l_{p}$ design technique for twodimensional-digital recursive filters," IEEE Trans. Acoust., Speech, Signal Processing, vol. ASSP-22, pp. 15-21, 1974.

[27] B. George and A. N. Venetsanopoulos, "Design of 2-D recursive digitl filters on the basis of quadrantal and octagonal symmetry," Circuits, Syst., Signal Processing, vol. 3, no. 1, pp. 59-78, Apr. 1984.

[28] N. A. Pendergrass, S. K. Mitra, and E. I. Jury, "Spectral transformations for two-dimensional digital filters," IEEE Trans. Circuits Syst., vol. CAS-23, pp. 26-35, 1976.

[29] R. E. Twogood and S. K. Mitra, "Computer aided design of separable two-dimensional digital filters," IEEE Trans. Acoust., Speech, Signal Processing, vol. ASSP-25, no. 2, pp. 165-169, 1977.

[30] J. L. Shanks, S. Treitel, and J. H. Justice, "Stability and synthesis of two-dimensional recursive filters," IEEE Trans. Audio Electroacoust., vol. AU-20, pp. 115-128, 1972.

[31] T. S. Huang, "Stability of two-dimensional recursive filters," IEEE Trans. Audio Electroacoust., vol. AU-20, pp. 158-163, 1972.

[32] N. K. Bose, "Implementation of a new stability test for two-dimensional filters," IEEE Trans. Acoust., Speech, Signal Processing, vol ASSP-25, pp. 117-120, 1977.

[33] G. Garibotto, "A new stability test for 2-D digital filters," Proc. Conf. EUSIPCO 1980, pp. 413-416, Lausanne, Switzerland, Sept. 1980.

[34] E. I. Jury, "Stability of multidimensional scalar and matrix polynomials," Proc. IEEE, vol. 66, pp. 1018-1047, 1978.

[35] M. G. Strintzis, "Tests of stability of multidimensional filters," IEEE Trans. Circuits Syst., vol. CAS-24, pp. 432-437, 1977.

[36] D. M. Goodman, "Some stability properties of 2-D linear shift-invariant digital filters," IEEE Trans. Circuits Syst., vol. CAS-24, pp. 201-208, 1977.

[37] P. Agathoklis, E. I. Jury, and M. Mansour, "The margin of stability of 2-D linear discrete systems," IEEE Trans. Acoust., Speech, Signal Processing, vol. ASSP-30, pp. 869-873, 1982.

[38] D. M. Goodman, "A design technique for circularly symmetry lowpass filters," IEEE Trans. Acoust., Speech, Signal Processing, vol. ASSP-26, pp. 290-304, 1978.

[39] B. G. Mertzios and A. N. Venetsanopoulos, "Noise properties of 2D IIR digital filters implemented by the decomposition theorem," in Proc. 2nd European Signal Processing Conference--(EUSIPCO-83), Erlangen, FRG, pp. 211-214, Sept. 1983.

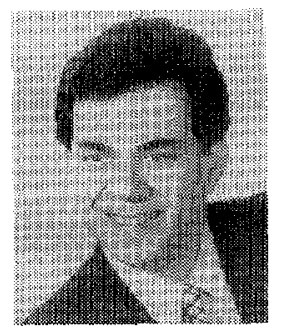

Anastasios N. Venetsanopoulos (S'66-M'69SM'79) received the mechanical and electrical engineering degree from the National Technical University of Athens, Greece, in 1965 . He received the M.S., M. Phil., and Ph.D. degrees from the Department of Engineering and Applied Science of Yale University in 1966, 1968, and 1969, respectively.

He joined the University of Toronto, Canada, in September 1968, where he is now Professor and Chairman of the Communications Group, Department of Electrical Engineering. His research interests span the fields of digital signal/image processing and digital communications. He has conducted research, taught, and consulted extensively in Europe, North and South America.

Dr. Venetsanopoulos is a member of the Association of Professional Engineers of Ontario the New York Academy of Sciences, Sigma Xi, and the Technical Chamber of Greece. He was Program Chairman of the International Communications Conference (ICC-78) and will be again for ICC-86. He has served as Chairman of the Central Canada Council of IEEE and the Editor of the Canadian Electrical Engineering Journal. He is presently a Fellow of the Engineering Institute of Canada, Associate Editor for Digital Signal Processing of the IEEE TRAnSACtions ON Circurrs AND SYSTEms, and the President of the Canadian Society for Electrical Engineering. 


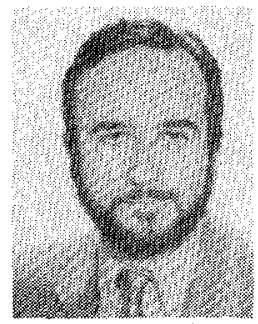

Basil G. Mertzios ( $\mathrm{S}^{\prime} 77-\mathrm{M}^{\prime} 82$ ) was born in Kavala, Greece, on August 10, 1956. He received the Diploma degree in electrical and mechanical engineering from the Aristotle University of Thessaloniki, Greece, in 1979, and the Ph.D. degree in electrical engineering from the Democritus University of Thrace, Greece, in 1982, both with honors.

From 1979 to 1981 he served as an officer in the Technical Corps of the Greek Army. During part of 1981 and 1983 he worked at the University

of Toronto, Department of Electrical Engineering, as a Research Assistant and a Postdoctoral Fellow, respectively. Since July 1982 he is a Lecturer at the Department of Electrical Engineering, Democritus University of Thrace, Greece. His major research interests are in the areas of analysis and control of multivariable and multidimensional systems, as well as of digital signal processing.

Dr. Mertzios is a member of the Technical Chamber of Greece. 